\title{
ON A MODIFIED FORM OF PURE RECIPROCANTS POSSESSING THE PROPERTY THAT THE ALGEBRAICAL SUM OF THE COEFFICIENTS IS ZERO
}

\author{
By Major P. A. MacMaHon, Sc.D., F.R.S.
}

[Received March 14th, 1914. - Read April 23rd, 1914.]

Prof. Sylvester at the close of his Sixteenth Lecture on the "Theory of Reciprocants," remarks:*-" It will be already seen from an inspection of the fundamental forms that there is no law for the coeffieients of reciprocants akin to that of their algebraical sum being zero in invariants."

I wish to shew in this short communication that the forms may be so modified that the vanishing of the algebraical sum of the coefficients is in evidence.

Denoting with Sylvester $d y / d x$ by $t$ and $d x / d y$ by $\tau$, also

$$
\frac{1}{(s+2) !} \frac{d^{s+2} y}{d x^{s+2}} \text { by } a_{s}, \quad \frac{1}{(s+2) !} \frac{d^{x+2} x}{d y^{s+2}} \text { by } a_{s} \text {, }
$$

we have the well known formulæ

$$
\begin{aligned}
a_{0} & =-a_{0} \div t^{3}, \\
a_{1} & =\left(-a_{1} t+2 a_{0}^{2}\right) \div t^{5}, \\
\alpha_{2} & =\left(-a_{2} t^{2}+5 a_{0} a_{1} t-5 a_{0}^{3}\right) \div t^{7}, \\
\alpha_{3} & =\left\{-a_{3} t^{3}+\left(6 a_{0} a_{2}+3 a_{1}^{2}\right) t^{2}-28 a_{0}^{2} a_{1} t+14 a_{0}^{4}\right\} \div t^{9}, \\
\alpha_{4}= & \left\{-a_{4} t^{4}+\left(7 a_{0} a_{3}+7 a_{1} a_{2}\right) t^{3}-\left(28 a_{0}^{2} a_{2}+28 a_{0} a_{1}^{2}\right) t^{2}\right. \\
& \left.+84 a_{0}^{3} a_{1} t-42 a_{0}^{5}\right\} \div t^{11},+\ldots .
\end{aligned}
$$

In these formula we may interchange the sets of symbols

$$
t_{1} a_{0}, a_{1}, \ldots, \quad \tau_{1} a_{0}, a_{1}, \ldots
$$

* Mathematical Papers, Vol. rv, p. 398.

† Observe that in the Mathematical Papers, Vol. rv, p. 311 , the term $28 a_{0} a_{1}^{\ddot{z}}$ is erroneously printed $28 a_{1}^{2}$, 
A pure reciprocant is a homogeneous and isobaric function of

$$
a_{0}, a_{1}, a_{2}, \ldots
$$

which is equal, the sign being disregarded, to the same function of

$$
a_{0}, a_{1}, \alpha_{2}, \ldots,
$$

multiplied by some power of $t$.

If we form from the above relations any such function of $a_{0}, \alpha_{1}, a_{2}, \ldots$, each term of the function, when expressed in terms of $t, a_{0}, a_{1}, a_{2}, \ldots$, gives rise to a term which is simply a power of $a_{0}$ multiplied by a power of $t$, and in the combination of terms which stands for a pure reciprocant these powers of $a_{0}$ must in combination vanish.

Ex. $g r \cdot$,

$$
\begin{aligned}
a_{v}^{2} \alpha_{3}-3 \alpha_{0} \alpha_{1} a_{2}+2 \alpha_{1}^{3}=\ldots+\left(-\frac{a_{0}}{t^{3}}\right)^{2} & \left(14 \frac{a_{0}^{4}}{t^{9}}\right) \\
& -3\left(-\frac{a_{0}}{t^{3}}\right)\left(2 \frac{a_{0}^{2}}{t^{5}}\right)\left(-5 \frac{a_{\mathrm{c}}^{3}}{t^{7}}\right)+2\left(2 \frac{a_{0}^{2}}{t^{5}}\right)^{3},
\end{aligned}
$$

and since the right-hand side is equal to $a_{0}^{2} a_{3}-3 a_{0} a_{1} a_{2}+2 a_{1}^{3}$ with a negative sign and a power of $t$, it is clear that the terms written on the right-hand side must vanish identically.

Hence, if $f\left(a_{0}, a_{1}, a_{2}, a_{3}, \ldots\right)$ be a pure reciprocant,

$$
f\left(-a_{0}, 2 a_{0}^{2},-5 a_{0}^{3}, 14 a_{0}^{4}, 42 a_{0}^{5}, \ldots\right)
$$

must vanish identically.

The general $a_{0}$ term here is

$$
(-)^{s} \frac{1}{s}\left(\begin{array}{c}
2 s \\
s-1
\end{array}\right) a_{0}^{*}
$$

Hence, if we write $a_{s}=(-)^{s+1} \frac{1}{s+1}\left(\begin{array}{c}2 s+2 \\ s\end{array}\right) b_{s}$,

that is to say

$$
b_{s}=(-)^{s+1} \frac{(s+1) !}{(2 s+2) !} \frac{d^{s+2} y}{d x^{s+2}},
$$

and denote the pure reciprocant by

$$
\phi\left(b_{0}, b_{1}, b_{2}, \ldots\right),
$$

the form is such that the algebraical sum of the coefficients vanishes.

If a term of the reciprocant be as regards the literal portion

$$
a_{0}^{\lambda_{0}} a_{1}^{\lambda_{1}} a_{2}^{\lambda_{2}} \ldots
$$


this, by the substitution, acquires the sign

$$
(-)^{\lambda_{0}+\lambda_{2}+\lambda_{1}+\ldots}
$$

which is the same for every term because

$$
(-)^{\lambda_{1}+\lambda_{2}+\lambda_{4}+\ldots}=(-)^{\left(\lambda_{0}+\lambda_{2}+\lambda_{2}+\ldots\right)-\left(\lambda_{1}+2 \lambda_{2}+3 \lambda_{3}+4 \lambda_{4}+\ldots\right)},
$$

and the right-hand side is constant for every term of the reciprocant. Hence we may take the substitution to be

$$
a_{s}=\frac{1}{s+1}\left(\begin{array}{c}
2 s+2 \\
s
\end{array}\right) b_{s},
$$

and then the algebraical sum of the coefficients vanishes. Otherwise we may assert that a pure reciprocant vanishes on putting

$$
a_{s}=\frac{1}{s+1}\left(\begin{array}{c}
2 s+2 \\
s
\end{array}\right) .
$$

The same result is deducible from the writer's theorem given in the Transactions of the Cambridge Philosophical Society, Vol. xxi, No. 6, pp. 143-170. The paper is entitled "The Operator Reciprocants of Sylvester's Theory of Reciprocants," and the theorem will be found on p. 166.

It states that the transformation

$$
\begin{aligned}
& a_{0}=\frac{1}{c_{0}}, \\
& a_{1}=-\frac{2 c_{1}}{c_{0}^{3}}, \\
& a_{2}=-\frac{3 c_{2}}{c_{0}^{4}}+\frac{8 c_{1}^{2}}{c_{0}^{5}}, \\
& a_{3}=-\frac{4 c_{3}}{c_{0}^{5}}+\frac{30 c_{1} c_{2}}{c_{0}^{6}}-\frac{40 c_{1}^{3}}{c_{0}^{7}}, \\
& \quad \& c .,
\end{aligned}
$$

converts all pure reciprocants in the elements

$$
a_{0}, a_{1}, a_{2}, a_{3}, \ldots,
$$

into seminvariants in the elements

$$
c_{0}, c_{1}, c_{2}, c_{3}, \ldots
$$

The reader will observe that on the dexter of the above relations the sums 
of the coefficients are respectively

$$
1,-2,+5,-14,+42, \ldots,(-)^{s} \frac{1}{s+1}\left(\begin{array}{c}
2 s+2 \\
s
\end{array}\right),
$$

and that knowing that in seminvariants the sum of the coefficients vanishes, we at once proceed to the theorem of this paper.

The substitution of

$$
\frac{1}{s+1}\left(\begin{array}{c}
2 s+2 \\
s
\end{array}\right) b_{s} \text { for } a_{s}
$$

in pure reciprocants leads to a remarkable simplification in the numerical values of the coefficients which must be of importance to any investigator who desires to study the mutual relations of the forms.

Below are given (1) Sylvester's forms, (2) the forms which arise by the modification which has been explained.

$$
\begin{gathered}
\left\{\begin{array}{l}
a_{0} \\
b_{0}
\end{array}\right. \\
\left\{\begin{array}{c}
4 a_{0} a_{2}-5 a_{1}^{2} \\
b_{0} b_{2}-b_{1}^{2}
\end{array}\right. \\
\left\{\begin{array}{c}
a_{0}^{2} a_{3}-3 a_{0} a_{1} a_{2}+2 a_{1}^{3} \\
7 b_{0}^{2} b_{3}-15 b_{0} b_{1} b_{2}+8 b_{1}^{3}
\end{array}\right. \\
\left\{\begin{array}{c}
50 a_{0}^{2} a_{4}-175 a_{0} a_{1} a_{3}+28 a_{0} a_{2}^{2}+105 a_{1}^{2} a_{2} \\
3 b_{0}^{2} b_{4}-\quad 7 b_{0} b_{1} b_{2}+\quad b_{0} b_{2}^{2}+\quad 3 b_{1}^{2} b_{2}
\end{array}\right. \\
\left\{\begin{array}{c}
800 a_{0}^{2} a_{2} a_{4}-875 a_{0}^{2} a_{3}^{2}-1000 a_{0} a_{1}^{2} a_{4}+2450 a_{0} a_{1} a_{2} a_{3}-1344 a_{0} a_{2}^{3}-35 a_{1}^{2} a_{2}^{2} \\
48 b_{0}^{2} b_{2} b_{4}-49 b_{0}^{2} b_{3}^{2}-\quad 48 b_{0} b_{1}^{2} b_{4}+\quad 98 b_{0} b_{1} b_{2} b_{3}-\quad 48 b_{0} b_{2}^{3}-\quad b_{1}^{2} b_{2}^{2}
\end{array}\right. \\
\left\{\begin{array}{c}
125 a_{0}^{3} a_{3}^{2}-750 a_{0}^{2} a_{1} a_{2} a_{3}+256 a_{0}^{2} a_{2}^{3}+165 a_{0} a_{1}^{2} a_{2}^{2}+500 a_{1}^{3} a_{3}-300 a_{1}^{4} a_{2} \\
49 b_{0}^{3} b_{0}^{2}-210 b_{0}^{2} b_{1} b_{2} b_{3}+64 b_{0}^{2} b_{2}^{3}+33 b_{0} b_{1}^{2} b_{2}^{2}+112 b_{1}^{3} b_{3}-48 b_{1}^{4} b_{2}
\end{array}\right. \\
\begin{array}{c}
625 a_{0}^{3} a_{4}^{2}-4375 a_{0}^{2} a_{1} a_{3} a_{4}-49700 a_{0}^{2} a_{2}^{2} a_{4}+55125 a_{0}^{2} a_{2} a_{3}^{2}+128625 a_{0} a_{1}^{2} a_{2} a_{4} \\
-61250 a_{0} a_{1}^{2} a_{3}^{2}-156800 a_{0} a_{1} a_{2}^{2} a_{3}+84868 a_{0} a_{2}^{4}
\end{array} \\
-78750 a_{1}^{4} a_{4}+183750 a_{1}^{3} a_{2} a_{3} \quad-102165 a_{1}^{2} a_{2}^{3}
\end{gathered}
$$

